assess whether this association can be explained by behavioural/ sexual networking patterns, the existence of a biological substrate, or both.

Disclosure of interest statement All authors declare no conflicts of interest.

\section{P11.19 SYPHILIS INCIDENCE AND ASSOCIATED RISK FACTORS AMONG MEN WHO HAVE SEX WITH MEN IN BANGKOK, THAILAND, 2006-2015}

${ }^{1} \mathrm{C}$ Ungsedhapand*, ${ }^{1} \mathrm{~S}$ Pattansin, ${ }^{1} \mathrm{~A}$ Sriporn, ${ }^{1} \mathrm{~S}$ Winaitham, ${ }^{1} \mathrm{~N}$ Promda, ${ }^{1} \mathrm{~W}$ Thienkrua, 'W Sukwicha, ${ }^{2} \mathrm{~A}$ Chitwarakorn, ${ }^{1,3} \mathrm{TH}$ Holtz, ${ }^{1,3} \mathrm{EF}$ Dunne. ${ }^{1}$ HIVISTD Research Program, Thailand Ministry of Public Health - US Centers for Disease Control and Prevention Collaboration, Nonthaburi, Thailand; ${ }^{2}$ Department of Disease Control, Ministry of Public Health, Nonthaburi, Thailand; ${ }^{3}$ Division of HIVIAIDS Prevention, US Centers for Disease Control and Prevention, Georgia, USA

\subsection{6/sextrans-2015-052270.467}

Introduction Syphilis infection has been increasing among men who have sex with men (MSM) in Thailand. We assessed syphilis incidence and associated risk factors in the Bangkok MSM Cohort Study (BMCS).

Methods We enrolled Thai MSM age $\geq 18$ years old in the BMCS from April 2006 to January 2008 (Period1) and September 2009 to December 2010 (Period2), and followed participants every 4 months for up to 60 months. Treponema pallidum (TP) screening was performed at enrollment, annually and at any unscheduled study visits if indicated, using the rapid plasma reagin (RPR). If the RPR was reactive, we confirmed with a TP-specific antibody test. We defined incident syphilis as a RPR titer $\geq 1: 8$ and a reactive treponemal test. We calculated risk factors for incident syphilis in all participants using a Cox proportional hazard model, adjusting for baseline demographic and behavioural characteristics, and prevalent HIV infection.

Results Among 1,502 participants who had no syphilis at baseline and had more than one follow-up visit, $54.7 \%$ reported unprotected anal intercourse (UAI). The overall syphilis incidence was 2.7 (95\% CI 2.3-3.1) per 100 person-years (/ $100 \mathrm{PY})$. There was a significant difference in syphilis incidence in the two periods $(2.5 / 100 \mathrm{PY}$ in Period1 vs. 3.3/100PY in Period2, $p=0.003$ ). Factors independently associated with incident syphilis were enrollment in Period2 (Adjusted HR [AHR] 1.7; 95\% CI 1.2-2.4), UAI (AHR 1.4; 95\% CI 1.0-2.0), amyl nitrate use (AHR 1.9; 95\% CI 1.2-2.8), Viagra use (AHR 1.6; 95\% CI 1.1-2.5), and prevalent HIV infection (AHR 2.3; 95\% CI 1.6-3.1).

Conclusion In the BMCS, statistically significant differences in incident syphilis by enrollment period may be due to recent increases in syphilis among urban Thai MSM. Syphilis screening identifies MSM at high risk for HIV, and syphilis screening and treatment are necessary for comprehensive HIV VCT services.

Disclosure of interest statement The Bangkok MSM Cohort Study (BMCS) is funded by the Thailand Minister of Public Health (MOPH) - US Centres for Disease Control and Prevention (CDC) Collaboration. No pharmaceutical grants were received in the development of this study.
P11.20 RESURGENCE OF SYPHILIS AMONG HIV-INFECTED MEN WHO HAVE SEX WITH MEN ATTENDING STI CLINICS IN THE NETHERLANDS

${ }^{1} \mathrm{~F}$ van Aar*\# ${ }^{1} \mathrm{C}$ den Daas $\#$, 1,2 MAB van der Sande, ${ }^{3} \mathrm{HJ}$ de Vries, ${ }^{1} \mathrm{BHB}$ van Benthem. ${ }^{1}$ Center for Infectious Disease Control, National Institute for Public Health and the Environment, Bilthoven, The Netherlands; ${ }^{2}$ Julius Center, University Medical Center, Utrecht, The Netherlands: ${ }^{3}$ Cluster of Infectious Diseases, Public Health Service Amsterdam, Amsterdam, The Netherlands; " These Authors Contributed Equally to This Work

\subsection{6/sextrans-2015-052270.468}

Introduction In the Netherlands, approximately $90 \%$ of all infectious syphilis (syphilis) and 70\% of all new HIV infections are among men who have sex with men (MSM). As the relation between syphilis and HIV is characterised by a bidirectional synergy, we assessed syphilis trends among MSM stratified by HIV status.

Methods The national STI/HIV surveillance contains epidemiological, behavioural and clinical data from STI clinics, which provide low threshold STI/HIV testing and care for high-risk groups. We descriptively examined syphilis positivity rates between 2007 and 2014.

Results The number of MSM consultations increased each year from 11,048 in 2007 to 29,939 in 2014. Of all MSM, $83 \%$ were HIV-negative. The number of syphilis diagnoses was lowest in $2011(\mathrm{n}=426)$ and highest in $2014(\mathrm{n}=693)$, of which around $40 \%$ was among HIV-infected MSM each year. Among known HIV-infected MSM, the syphilis positivity rate decreased between $2007(12.3 \%)$ and 2011 (4.5\%), followed by an increase in recent years (2014: 6.6\%). Among MSM newly diagnosed with HIV, this trend was roughly similar to that of known HIV-infected MSM. Among HIV-negative MSM, the syphilis positivity rate decreased between 2007 (2.8\%) and 2011 (1.4\%), and remained stable thereafter.

Conclusion The observed rebound in syphilis rates in a high-risk group of HIV-positive MSM is concerning at individual level, given the adverse effects on both syphilis and HIV disease progression. At public health level, this is worrisome as syphilis facilitates transmission and acquisition of HIV. Therefore, syphilis infection could be indicative to offer HIV pre-exposure prophylaxis (PrEP) among HIV-negative MSM. On the other hand, offering PrEP to a high-risk core group could promote syphilis transmission if it leads to reduced perceived vulnerability and subsequent increased risk behaviour, as seen after the introduction of cART.

Disclosure of interest statement The authors declare that they have no competing interests.

\section{P11.21 CHARACTERISING THE EPIDEMIOLOGY OF ACTIVE HEPATITIS B VIRUS INFECTION AMONG HIGH-RISK MEN WHO HAVE SEX WITH MEN AND TRANSGENDER WOMEN IN LIMA, PERU}

${ }^{1} \mathrm{MC}$ Herrera*, ${ }^{1} \mathrm{KA}$ Konda, ${ }^{2} \mathrm{SR}$ Leon, ${ }^{3} \mathrm{PA}$ Wong, ${ }^{4} \mathrm{~B}$ Brown, ${ }^{2} \mathrm{CF}$ Caceres, ${ }^{1} \mathrm{JD}$ Klausner. ${ }^{1}$ Division of Infectious Diseases, UCLA David Geffen School of Medicine, Los Angeles, California, USA; ' Laboratory of Sexual Health, and Unit of Health, Sexuality and Human Development, Universidad Peruana Cayetano Heredia, Lima, Peru; ${ }^{3}$ Instituto Peruano de Investigaciones en Biotecnología, Lima, Peru; ${ }^{4}$ Department of Population Health \& Disease Prevention, UC Irvine, Irvine, California, USA

10.1136/sextrans-2015-052270.469 